\title{
Projeto HistoQuiz: Desenvolvendo um Jogo Voltado para o Ensino de Histologia
}

\author{
Marcella A. P. Bastos ${ }^{1}$, Maria da C. Andrade ${ }^{1}$, \\ Victória O. Gomes ${ }^{1}$, Victor T. Sarinho ${ }^{1}$ \\ ${ }^{1}$ Laboratório de Entretenimento Digital Aplicado - LEnDA \\ Universidade Estadual de Feira de Santana - UEFS \\ Feira de Santana - Bahia - Brasil \\ marcellaaraujo99@gmail.com, conceicao.icb@gmail.com \\ victoria.oliveiragomes@gmail.com, vsarinho@uefs.br
}

\begin{abstract}
The teaching of histology requires contact of students with histological slides, which occurs basically in practical microscopy classes. This contact is limited to the class time, which is often reduced, and the acquisition of slides from different organs that must be made available for individual observation, whose quantity and variety is also reduced. The absorption of this content can be facilitated with the use of Information and Communication Technologies (ICTs), which are tools of easy access and continuous use, thus providing motivation, participation and interaction with this material. Thus, in order to promote a way of exercising such matters better, this article elucidates the steps followed for the development of HistoQuiz, a digital mobile game created to transmit knowledge related to histology.
\end{abstract}

Resumo. $O$ ensino de histologia requer o contato dos alunos com lâminas histológicas, o que ocorre basicamente nas aulas práticas de microscopia. Esse contato fica limitado ao tempo da aula, que muitas vezes é reduzido, e a aquisição de lâminas de diversos órgãos que devem ser disponibilizadas para a observação individual, cuja quantidade e variedade também é reduzida. A absorção desse conteúdo pode ser facilitada com o uso das Tecnologias de Informação e Comunicação (TICs), que constituem ferramentas de fácil acesso e uso contínuo, propiciando assim motivação, participação e interação com esse material. Buscando promover uma maneira de exercitar, o presente artigo elucida as etapas seguidas para o desenvolvimento do HistoQuiz, um jogo digital mobile criado para transmitir conhecimentos relacionados a histologia.

\section{Introdução}

A histologia (do grego hystos $=$ tecido $+\operatorname{logos}=$ estudo), também chamada de anatomia microscópica, é o estudo científico de estruturas microscópicas de tecidos e órgãos do corpo. A histologia moderna inclui aspectos de biologia molecular e celular, que ajudam a descrever a organização e a função das células. Boa parte do conteúdo do curso de histologia é estruturado na microscopia óptica. Atualmente nos laboratórios de histologia os estudantes usam os microscópios de luz ou, com uma frequência crescente, a microscopia virtual, que representa um método de observação 
de espécimes microscópicos digitalizados em uma tela de computador ou dispositivos móveis [Ross and Pawlina 2012].

Tal ciência compreende uma das maiores esferas de ensino laboratorial pertencentes ao currículo de cursos voltados para o estudo do organismo humano, onde, por muito tempo, o microscópio foi o principal instrumento de ensino utilizado [Bloodgood and Ogilvie 2006]. Essa prática, no entanto, vem sendo modificada nos últimos anos, com a inserção da tecnologia como auxiliadora no processo de transmissão desse conhecimento [Cotter 2001], fazendo com que um número reduzido de horas sejam dedicadas à instrução puramente laboratorial.

Atualmente vivemos em uma sociedade com grande desenvolvimento tecnológico, o qual tem causado modificações nos diversos setores, inclusive no âmbito escolar. As Tecnologias da Informação e Comunicação (TICs) têm despertado a necessidade de um novo paradigma de ensino, voltado para o estudante e na promoção de estratégias que possibilitem torná-lo um indivíduo com capacidade de posicionamento. Nesse cenário, o papel do docente foi redefinido e a sua missão deixou de ser, apenas, ensinar sobre um determinado material, mas ensinar a aprender [Vieira et al. 2014].

Apesar da aquisição de conhecimento baseada na utilização de práticas pedagógicas tecnológicas ser algo raramente encontrado no campo da medicina [Ackermann et al. 2006], no ramo da histologia é possível obter grandes vantagens a partir do seu uso. Isso se deve ao fato de que o estudo nessa disciplina é baseado em "terminologias, descrições de morfologia, figuras e desenhos que devem ser memorizados" [Ackermann et al. 2006], o que depende da utilização de uma série de equipamentos específicos que permitam a visualização de células e sua organização nos diversos tecidos do corpo e que, muitas vezes, não estão disponíveis para os estudantes por um longo período de tempo.

Se tratando de histologia, afim de consolidar o conhecimento obtido faz-se necessário horas de observação e repetição de estudo desse conteúdo [de Oliveira Júnior and da Silva 2014]. Nesse âmbito, as TICs podem ser utilizadas para proprocionar a possibilidade de rever conteúdos que só poderiam ser observados com aparelhos específicos como, por exemplo, os microscópios [Santa-Rosa and Struchiner 2011].

Assim, levando em conta o fato de que, ao ter acesso pela primeira vez ao conteúdo abrangido pela histologia, por vezes, os alunos podem apresentar dificuldades devido à complexidade da matéria ou ao tempo insuficiente de permanência no laboratório [de Oliveira Júnior and da Silva 2014], este trabalho descreve o desenvolvimento do HistoQuiz, um jogo mobile voltado para o ensino e o exercício de conteúdos de histologia.

\section{Trabalhos Relacionados}

A utilização de objetos tecnológicos voltados para o ensino e aprendizagem de histologia vem se mostrando muito eficaz. Isso se deve ao fato de que as TICs proporcionam a utilização de práticas pedagógicas interativas e simples que podem ser empregadas pelos estudantes para maximizar a absorção de conhecimentos relacionados ao assunto, além de conseguir captar de maneira mais poderosa a atenção do docente 
[dos Santos Bardini et al. 2017]. Como resultado, diferentes trabalhos vem apresentando o uso das TICs no ensino da histologia, a partir do desenvolvimento e utilização de ambientes virtuais de apredizagem (AVE) [Vasconcelos and Vasconcelos 2013] [Santa-Rosa and Struchiner 2010].

Com relação ao desenvolvimento de jogos digitais para o ensino de histologia, pode-se apresentar os resultados obtidos com a aplicação de um jogo estilo quiz para o ensino de histologia baseado na plataforma Kahoot [Rojas-Mancilla et al. 2019]. Um outro exemplo de jogo digital desenvolvido é o Histology Card! [Bauman et al. 2017], no qual os jogadores recebem uma placa de imagem histológica a qual precisam complementar com imagens apresentadas em cartões histológicos correspondentes.

\section{Metodologia}

A dinâmica do HistoQuiz acontece da seguinte forma: no início, três lâminas são sorteadas para cada jogador, as quais deverão ser adivinhadas pelo seu oponente. Vence aquele que desvendar o conteúdo de cada uma das três lâminas primeiro. Para obter dicas relacionadas a esse conteúdo, a cada jogada será possível fazer uma pergunta a seu adversário, cuja resposta sempre se limitará a "sim" ou "não", a fim de obter uma nova informação sobre o que se está tentando adivinhar. As perguntas disponíveis são exibidas em separadas por categorias, e o jogador deverá escolher aquela cuja resposta ele acredita que fornecerá uma melhor pista sobre a lâmina que está tentando adivinhar.

Com o objetivo de armazenar e analizar dados relacionados ao desempenho do usuário no jogo, é necessário que o jogador se cadastrar no jogo antes de começar seu uso, fornecendo algumas informações como nome, email e senha. Em relação as modalidades de jogo, este apresenta a possibilidade de jogar contra o dispositivo ou jogar contra outro amigo. Para isso, o jogo dispõe de uma seção para o gerenciamento de conta, onde é possível enviar convite, remover e aceitar amigos, além de editar seus dados pessoais e verificar seu desempenho nas partidas jogadas, criando assim uma espécie de rede social para os jogadores do jogo.

Com relação as imagens das lâminas e as perguntas relacionadas a elas, assim como os dados de cadastro de cada um dos usuários do jogo, todos são armazenados no Firebase, uma plataforma que funciona como uma espécie de servidor e banco de dados para aplicações web e mobile. Atualmente, existem 50 lâminas disponíveis no jogo, e um total de 143 perguntas, separadas em sete categorias diferentes, para auxiliar o jogador a desvendá-las. Todos esses dados foram obtidos em conjunto com uma professora da área de histologia e uma aluna do curso de medicina, visando embasá-los corretamente.

Em relação a plataforma de execução do jogo, o HistoQuiz foi desenvolvido para dispositivos Android através do Godot (versão 3.2), uma engine de código aberto que possui uma grande quantidade de recursos para o desenvolvimento de jogos mobile $2 \mathrm{D}$ e 3D conectados a serviços web desejados.

\section{Resultados e Discussão}

Por ser voltado ao ensino de histologia, uma área voltada para o estudo de tecidos e células, optou-se por utilizar no jogo um design que lembrasse remotamente a visualização obtida ao se olhar por um microscópio. Assim, ao abrir o app do jogo, o 
usuário tem a possibilidade de criar uma conta, ou, caso já tenha se registrado, fazer um login. A partir daí, o jogador será automaticamente redirecionado para a tela da Figura 1(a), onde poderá escolher entre verificar as informações da sua conta, jogar, revisar alguns assuntos relacionados aos possíveis conteúdos das lâminas ou visualizar informações sobre o jogo.

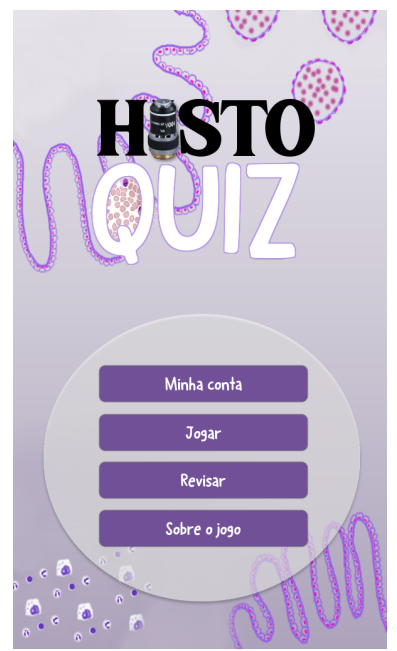

(a) Menu inicial.

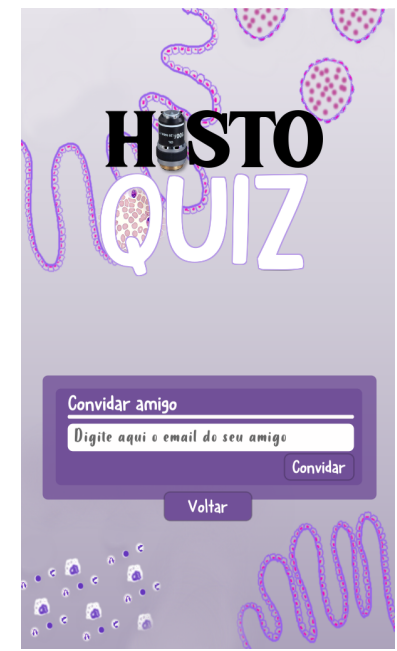

(b) Tela de convidar amigo.

Figure 1. Tela de login e de menu inicial do HistoQuiz.

Caso opte por jogar, o usuário deverá escolher a modalidade que deseja: jogar online ou jogar contra o computador. Ao escolher jogar online, o jogador deverá convidar um adversário para jogar, conforme a tela mostrada na Figura 1(b). Após o jogador convidado aceitar a solicitação, a tela de jogo da Figura 2(a) será exibida.

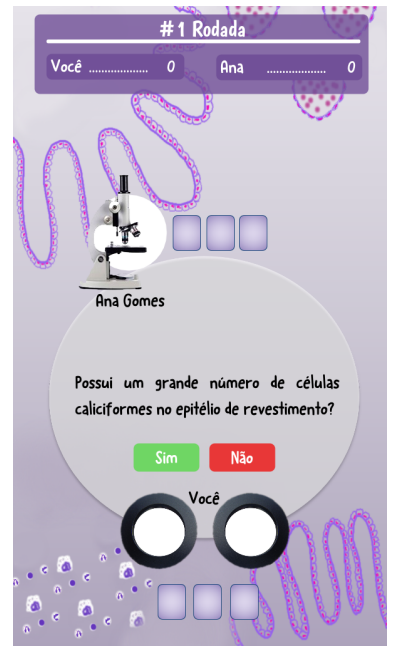

(a) Tela de jogo.

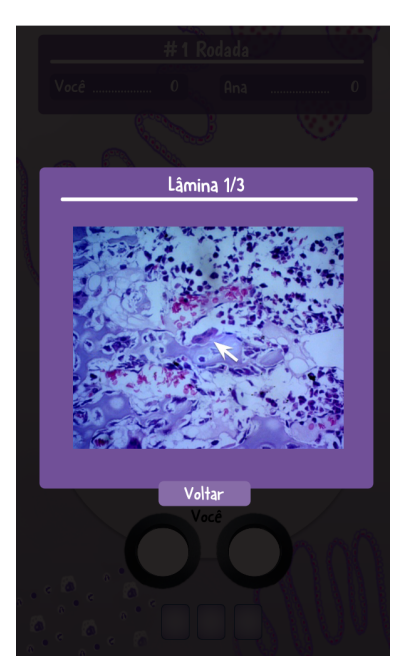

(b) Visualização da lâmina do oponente.

Figure 2. Tela de convidar amigo e de jogo do HistoQuiz.

A partida é constituída por dois jogadores, onde cada um dispõe de três lâminas para serem adivinhadas pelo seu adversário, e aquele que conseguir decifrar todas as 
lâminas primeiro vence. Uma visualização da lâmina do adversário pode ser observada na Figura 2(b). Para conseguir fornecer as respostas corretas, o jogador terá o auxílio de algumas perguntas. A cada rodada um jogador: 1) seleciona uma pergunta dentre as disponíveis para sua lâmina, que são relacionadas a características particulares dela; 2) seu oponente a responde, visto que ele tem acesso a lâmina que se está tentando adivinhar; e, em seguida, 3) o jogo revela o gabarito correto. Caso o oponente tenha acertado, 2 pontos são adicionados no seu placar automaticamente. Caso erre, ele deve perder 1 ponto. Após cada pergunta respondida pelo oponente, o jogador tem a opção de tentar acertar sua lâmina e ganhar mais pontos, estando assim mais próximo de vencer o jogo (adivinhar as 3 lâminas) ou passar a vez e aguardar a jogada do seu adversário para, posteriormente, fazer mais questionamentos.

A fim de ganhar o jogo, com base nas características obtidas a cada resposta, o jogador deverá analisá-las em conjunto e, a partir daí, inferir qual tecido está presente na sua lâmina. Cada jogador só terá acesso a segunda lâmina após a primeira ter sido adivinhada e assim por diante.

Afim de estimular a competitividade e, ao mesmo tempo, proporcionar um feedback para o usuário acerca do seu desempenho em tópicos específicos relacionados a histologia, é possível verificar seu progresso na tela exibida na Figura 3(a). Caso deseje obter mais conhecimentos antes de jogar, é possível fazer uma revisão relacionada ao assunto de sua escolha, como observado da Figura 3(b).

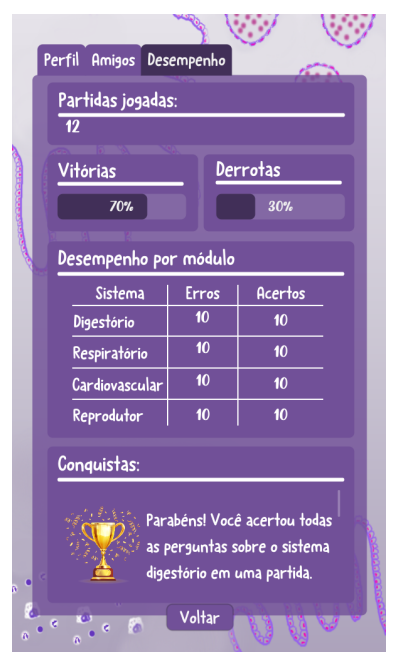

(a) Tela de desempenho.

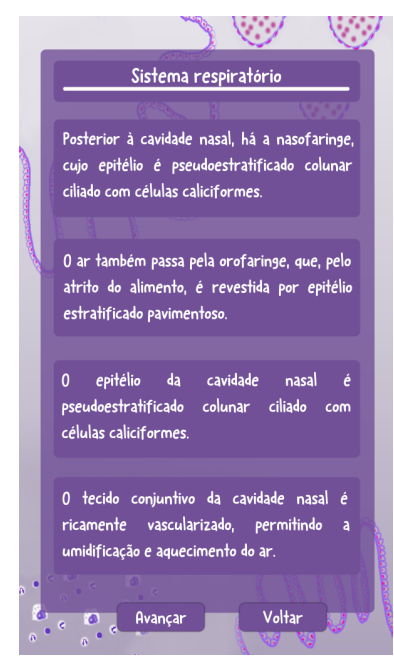

(b) Tela de revisar assunto.

Figure 3. Tela de desempenho de usuário e de revisão do HistoQuiz.

\section{Conclusões e Trabalhos Futuros}

O presente artigo descreveu o desenvolvimento do HistoQuiz, um jogo mobile voltado para a disseminação de conhecimentos relacionados a histologia. Trata-se de uma ferramenta importante que pode ser utilizada para diminuir a dificuldade associada ao primeiro contato dos estudantes com os conteúdos de tal disciplina.

Como trabalhos futuros, almeja-se prosseguir com o desenvolvimento de novas modalidades de jogo, como possibilitar a batalha entre duplas, obtendo assim um jogo 
colaborativo competitivo. Além disso, a quantidade de questões e lâminas presentes no banco de dados será aumentada, a fim de promover uma maior variedade de possibilidade de partidas. Antes de aplicar tais melhorias, no entanto, uma avaliação da efetividade e aceitação do jogo será efetuada ao disponibilizá-lo para uso de alguns estudantes de medicina, juntamente com o apoio da professora de histologia e da aluna que fazem parte do projeto, de modo a obter feedbacks mais sólidos de melhorias a serem aplicadas.

\section{References}

Ackermann, P. C. et al. (2006). The suitability of a multimedia resource for teaching undergraduate histology in a developing country. $\mathrm{PhD}$ thesis, University of Pretoria.

Bauman, E. B., Gilbert, G. E., and Vaughan, G. (2017). Short-term gains in histology knowledge: a veterinary gaming application. PeerJ Preprints.

Bloodgood, R. A. and Ogilvie, R. W. (2006). Trends in histology laboratory teaching in united states medical schools. The Anatomical Record Part B: The New Anatomist, 289(5):169-175.

Cotter, J. R. (2001). Laboratory instruction in histology at the university at buffalo: recent replacement of microscope exercises with computer applications. The Anatomical Record: An Official Publication of the American Association of Anatomists, 265(5):212-221.

de Oliveira Júnior, J. K. and da Silva, M. A. D. (2014). As tecnologias de informação e comunicação como ferramenta complementar no ensino da histologia nos cursos odontologia da região norte. Journal of Health Informatics, 6(2).

dos Santos Bardini, V. S., Spalding, M., Vasconcelos, L., Silveira, V., and Salgado, M. A. (2017). Práticas pedagógicas no ensino de histologia: estratégias para incentivar o aluno na consolidação dos conhecimentos. Revista Brasileira de Ensino Superior, 2(4):15-21.

Rojas-Mancilla, E., Conei, D., Bernal, Y. A., Astudillo, D., and Contreras, Y. (2019). Learning histology through game-based learning supported by mobile technology. International Journal of Morphology, 37(3).

Ross, M. H. and Pawlina, W. (2012). Histologia texto e atlas-em correlação com biologia celular e molecular.

Santa-Rosa, J. G. and Struchiner, M. (2010). Design participativo de um ambiente virtual de aprendizagem de histologia. Revista Brasileira de Pesquisa em Educação em Ciências, 10(2).

Santa-Rosa, J. G. and Struchiner, M. (2011). Tecnologia educacional no contexto do ensino de histologia: pesquisa e desenvolvimento de um ambiente virtual de ensino e aprendizagem. Revista Brasileira de Educação Médica, 35(2):289-298.

Vasconcelos, D. F. P. and Vasconcelos, A. C. C. G. (2013). Desenvolvimento de um ambiente virtual de ensino em histologia para estudantes da saúde. Revista Brasileira de Educação Médica, 37(1):132-137.

Vieira, F., Restivo, M. T., et al. (2014). Novas tecnologias e educação: Ensinar a aprender, aprender a ensinar. Biblioteca Digital da Faculdade de Letras da Universidade do Porto. 